\title{
VITA: FRIEDRICH WILHELM WIENER
}

\author{
HAROLD P. BOAS AND DMITRY KHAVINSON
}

\begin{abstract}
We report on the life and work of F. Wiener, whom we confused with $\mathrm{N}$. Wiener in a previous article.
\end{abstract}

\section{INTRODUCTION}

In a recent note [4], we proved a multi-dimensional analogue of the following classical theorem of Harald Bohr [6]. (For subsequent developments in the multi-dimensional theory, see [3, Q, 目.)

Theorem 1 (Bohr). Suppose that a power series $\sum_{k=0}^{\infty} c_{k} z^{k}$ converges for $z$ in the unit disk, and $\left|\sum_{k=0}^{\infty} c_{k} z^{k}\right|<1$ when $|z|<1$. Then $\sum_{k=0}^{\infty}\left|c_{k} z^{k}\right|<1$ when $|z|<1 / 3$. Moreover, the radius $1 / 3$ is the best possible.

In one part of the proof, we adapted to higher dimensions an elegant argument that Bohr attributed to Wiener. Since Bohr mentioned this name in the same sentence with the names of Riesz and Schur, we assumed it to be the famous Norbert Wiener, and we added the initial "N" in our attribution. Our assumption was false. Lawrence Zalcman brought to our attention that Edmund Landau mentioned the name of one F. Wiener in connection with Bohr's theorem [9, §4].

\section{WiEnER's LIFE}

Having never heard of a mathematician F. Wiener, we investigated. We report here on what information we have discovered about the life and work of F. Wiener, hoping that his name may be preserved in mathematical history for another generation.

According to the curriculum vitae accompanying his dissertation, Friedrich Wilhelm Wiener was born in 1884 in Meseritz, then part of the Prussian province of Posen and now part of Poland. After completing high school (gymnasium), he pursued studies in Göttingen. After a

1991 Mathematics Subject Classification. Primary 30-01; secondary 01A70, 26D15, 30D15.

The authors' research was partially supported by grants from the National Science Foundation. 
year of compulsory military service in 1904-1905, he resumed studies in Berlin. He returned to Göttingen in 1909, the same year that Landau was called there as Minkowski's successor. Wiener attended lectures of such famous mathematicians as Frobenius, Hilbert, Landau, Schottky, Schur, and Schwarz. He completed his doctoral dissertation [15] under the supervision of Landau in 1911.

Wiener published one journal article [14] in 1910, which is cited in standard books [7, 11]. After a promising beginning, he seems to have published nothing further, not even his dissertation. There is no evidence that Wiener was ever a member of the Deutsche MathematikerVereinigung (DMV); no obituary notice for Wiener appeared in the DMV Jahresbericht [10]. Although we do not know the circumstances of Wiener's death, this must have occurred no later than 1921, as the index published that year to volumes 51-80 of Mathematische Annalen lists Wiener as deceased. We conjecture that Wiener may have been a casualty of the war.

\section{WIENER'S WORK}

The focus of Wiener's mathematical work was to discover simple proofs of known theorems. Both of his papers have the word "elementary" in the title.

3.1. Hilbert's inequality. Wiener's 1910 paper concerns Hilbert's double series theorem stating the boundedness in $\ell_{2}$ of the quadratic form $\sum_{m=1}^{\infty} \sum_{n=1}^{\infty} x_{m} x_{n} /(m+n)$.

Theorem 2 (Hilbert).

$$
\left|\sum_{m=1}^{\infty} \sum_{n=1}^{\infty} \frac{x_{m} x_{n}}{m+n}\right| \leq C \sum_{n=1}^{\infty}\left|x_{n}\right|^{2} .
$$

Moreover, the inequality holds with $C=\pi$, and no smaller value of the constant $C$ will do.

Hilbert's proof was first published in the dissertation [13 of his student Hermann Weyl in 1908. The theorem attracted a great deal of attention, and numerous proofs and generalizations were published subsequently. The classical book by Hardy, Littlewood, and Pólya [0] devotes a whole chapter to this inequality. At the time of Wiener's work, it was not known that the sharp value of the constant $C$ is $\pi$ : Schur proved this the following year [12].

What Wiener meant by an "elementary" proof of Hilbert's inequality was a proof that used no integration and no function theory. His proof consists of the following elementary steps. 
1. Reduce to the case that $\left\{x_{n}\right\}_{n=1}^{\infty}$ is a decreasing sequence of positive real numbers.

2. Group the terms in the inner sum into blocks whose terms have indices running between consecutive squares.

3. Apply the Cauchy-Schwarz inequality to both the inner sum and the outer sum.

4. Interchange the order of summation.

5. Invoke Cauchy's condensation test for convergence of series.

3.2. Wiener's dissertation. In his dissertation, Wiener addresses two questions in the theory of entire functions of one complex variable.

The first part of the dissertation concerns the minimum modulus of an entire function $f$. Let $m(r)=\min \left\{\left|f\left(r e^{i \theta}\right)\right|: 0 \leq \theta \leq 2 \pi\right\}$. Since $m(r)$ is zero when $f$ has a zero of modulus $r$, the natural question to ask about a lower bound for $m(r)$ is whether $m(r)$ is frequently large: is there some reasonable comparison function $c(r)$ such that $\lim \sup _{r \rightarrow \infty} m(r) / c(r)>0$ ?

If $f$ is an entire function of finite order at most $\rho$, meaning that $\lim _{|z| \rightarrow \infty}|f(z)| e^{-|z|^{\rho+\epsilon}}=0$ for every positive $\epsilon$, then Hadamard's factorization theorem implies that $\limsup _{r \rightarrow \infty} m(r) e^{r^{\rho+\epsilon}}=\infty$ for every positive $\epsilon$. In other words, $m(r)$ cannot tend to zero too fast. This weak estimate cannot be improved in general. For example, the exponential function $e^{z}$ has order 1 and $m(r)=e^{-r}$. On the other hand, if $f$ is a non-constant polynomial, then $m(r)$ tends to infinity like a power of $r$. The question arises of whether an entire function of sufficiently small order is enough like a polynomial that its minimum modulus must be unbounded.

In 1905, A. Wiman confirmed [16 that the minimum modulus of every non-constant entire function of order $\rho$ strictly less than $1 / 2$ is indeed unbounded. Moreover, $\limsup _{r \rightarrow \infty} m(r) e^{-r^{\rho-\epsilon}}=\infty$ when $0<\epsilon<\rho<1 / 2$. The cutoff at $1 / 2$ is sharp, for the convergent infinite product $\prod_{n=1}^{\infty}\left(1-\frac{z}{n^{2}}\right)$, which equals $(\sin \pi \sqrt{z}) /(\pi \sqrt{z})$, has order $1 / 2$ and $m(r) \leq r^{-1 / 2} / \pi$. (See [5, Chapter 3] for more about the minimum modulus of entire functions of small order.)

Wiener's dissertation gives a new proof of Wiman's theorem. The proof is elementary in the sense that it uses only arguments about series and products of real numbers; it avoids using theorems from function theory.

Wiener's proof even supplements Wiman's theorem by giving some information in the endpoint cases $\rho=0$ and $\rho=1 / 2$. Namely, Wiener 
shows that if $f(z)=\prod_{n=1}^{\infty}\left(1-\frac{z}{a_{n}}\right)$, where $\left\{a_{n}\right\}_{n=1}^{\infty}$ is a sequence of nonzero complex numbers of increasing modulus, and if $\lim _{n \rightarrow \infty} n^{2} /\left|a_{n}\right|=$ 0 , then $\limsup _{r \rightarrow \infty} m(r) r^{-k}=\infty$ for every positive $k$. This result applies to all transcendental entire functions of order 0 (for example, to $\left.\prod_{n=1}^{\infty}\left(1-\frac{z}{n^{n}}\right)\right)$ and to some entire functions of order $1 / 2$ (for example, to $\left.\prod_{n=2}^{\infty}\left(1-\frac{z}{n^{2} \log n}\right)\right)$.

The second part of Wiener's dissertation is motivated by a theorem of Landau [8] that generalizes Picard's little theorem.

Theorem 3 (Landau). There is a positive function $R$ such that every polynomial of the form $a_{0}+z+a_{2} z^{2}+\cdots+a_{n} z^{n}$ assumes at least one of the values 0 and 1 in the disk $\left\{z:|z| \leq R\left(a_{0}\right)\right\}$. The function $R$ is independent of the degree $n$ and the higher coefficients $a_{2}, \ldots, a_{n}$.

One might hope that a theorem about polynomials would have an elementary proof, which would then yield an elementary proof of Picard's theorem. Wiener was able to find an elementary proof (using Rouché's theorem, but nothing else from function theory) of Landau's theorem under an additional hypothesis about the location of the zeroes of the polynomial. Namely, he assumed that the zeroes are located within the two equal acute angles determined by two lines intersecting at the origin. If the radian measure of the acute angle is $\frac{1}{2} \pi-\beta$, then one can take $R\left(a_{0}\right)=28\left|a_{0} \log a_{0}\right| / \sin \beta$. (The cases $a_{0}=0$ and $a_{0}=1$ are of no concern, because then the polynomial takes the value 0 or 1 at the origin.)

\section{ACKNowledgments}

For assistance in this project of identifying and tracing F. Wiener, we thank Samuel J. Patterson (Georg-August-Universität Göttingen), Constance Reid, Heinrich Wefelscheid (Gerhard-Mercator-Universität Gesamthochschule Duisburg), and Lawrence A. Zalcman (Bar Ilan University). We are especially indebted to Professor Wefelscheid for locating and sending to us a copy of Wiener's dissertation. We thank Heidemarie Wörmann Boas for help with German translation.

\section{REFERENCES}

1. L. Aizenberg, A. Aytuna, and P. Djakov, An abstract approach to Bohr's phenomenon, 1998 preprint.

2. __ Generalization of Bohr's theorem for arbitrary bases in spaces of holomorphic functions of several variables, 1998 preprint.

3. Lev Aizenberg, Multidimensional analogues of Bohr's theorem on power series, to appear in Proc. Amer. Math. Soc., math.CV/9804102. 
4. Harold P. Boas and Dmitry Khavinson, Bohr's power series theorem in several variables, Proc. Amer. Math. Soc. 125 (1997), no. 10, 2975-2979, math.CV/9606203.

5. Ralph P. Boas, Entire functions, Academic Press, 1954.

6. Harald Bohr, A theorem concerning power series, Proc. London Math. Soc. (2) 13 (1914), 1-5.

7. G. H. Hardy, J. E. Littlewood, and G. Pólya, Inequalities, Cambridge University Press, 1934, second edition 1952.

8. Edmund Landau, Über eine Verallgemeinerung des Picardschen Satzes, Sitzungsberichte der Königlich Preussischen Akademie der Wissenschaften (1904), 1118-1133.

9. _— Darstellung und Begründung einiger neuerer Ergebnisse der Funktionentheorie, Springer, 1916, second edition 1929, third edition 1986 edited and supplemented by Dieter Gaier.

10. Deutsche Mathematiker-Vereinigung, Nachrufe, an index of the obituary notices from the DMV Jahresbericht is available on the World-Wide Web at the address http://www.mathematik.uni-bielefeld.de/DMV/archiv/ nachrufe.html.

11. G. Pólya and G. Szegö, Problems and theorems in analysis, vol. I, Springer, 1972.

12. I. Schur, Bemerkungen zur Theorie der beschränkten Bilinearformen mit unendlich vielen Veränderlichen, J. Reine Angew. Math. 140 (1911), 1-28.

13. Hermann Weyl, Singuläre Integralgleichungen mit besonderer Berücksichtigung des Fourierschen Integraltheorems, Ph.D. thesis, Göttingen, 1908.

14. F. Wiener, Elemetarer Beweis eines Reihensatzes von Herrn Hilbert, Math. Ann. 68 (1910), 361-366.

15. Friedrich Wilhelm Wiener, Elementare Beiträge zur neueren Funktionentheorie, Ph.D. thesis, Georg-August-Universität, Göttingen, 1911.

16. A. Wiman, Sur une extension d'un théorème de M. Hadamard, Arkiv för Mathematik, Astronomi och Fysik 2 (1905), no. 14, 1-5.

Department of Mathematics, Texas A\&M University, College StaTION, TX 77843-3368

E-mail address: boas@math.tamu.edu

Department of Mathematical Sciences, University of Arkansas, FayetTEVILLE, AR 72701

E-mail address: dmitry@comp.uark.edu 